\title{
Effect of immunisation against vasoactive intestinal polypeptide on gastric corpus tone and motility in the ferret
}

\author{
D Grundy, M K Gharib-Naseri, D Hutson
}

\begin{abstract}
The role of vasoactive intestinal polypeptide in the control of gastric corpus tone and motility was investigated using auto-antibodies to neutralise endogenous vasoactive intestinal polypeptide. Six ferrets were immunised with vasoactive intestinal polypeptide thyroglobulin conjugate in Freund's complete adjuvant which resulted in a significant increase in plasma vasoactive intestinal polypeptide binding activity compared with unimmunised control animals. In acute experiments the level of spontaneous motility in the period immediately after completion of the surgical preparation was 15 times higher in immunised $v$ control animals $(\mathbf{p}<\mathbf{0 . 0 2})$. Surprisingly, however, there was no deficit in the ability of the corpus to accommodate fluid. Peak pressure at the end of a $20 \mathrm{ml}$ ramp distension was not different in immunised animals $\left(5.7(0.6) \mathrm{cm} \mathrm{H}_{2} \mathrm{O}\right)$ compared with controls $\left(4 \cdot 8(0 \cdot 3) \mathrm{cm} \mathrm{H}_{2} \mathrm{O}\right)$. It is concluded that the non-adrenergic non-cholinergic inhibitory mechanisms regulating corpus tone and motility are different and that vasoactive intestinal polypeptide acts primarily to regulate phasic contractile activity. Alternatively, because of plasticity in the mechanisms controlling corpus tone, the effect of vasoactive intestinal polypeptide may have been superceded during the timecourse of the immunisation procedure.

(Gut 1992; 33: 1473-1476)
\end{abstract}

The stomach receives extensive innervation from sympathetic and parasympathetic nerves. ${ }^{1}$ Both pathways can mediate inhibition of gastric motor function. The former follows the release of noradrenaline from postganglionic fibres acting predominantly at the level of the enteric nervous system. The latter is mediated by preganglionic vagal fibres activating postganglionic elements in the gut wall which utilise a non-adrenergic noncholinergic transmitter.

The vagal inhibitory mechanisms operate under physiological conditions such as gastric accommodation. ${ }^{2}$ In contrast, sympathetic inhibition is generally considered to dominate under pathophysiological conditions except at sphincters. ${ }^{3}$ In this respect the suppression of gastric motility seen after abdominal surgery has been considered principally to be a sympathetically mediated phenomenon. Some success in the treatment of postoperative ileus with sympatholytic agents has been reported ${ }^{4}$ but treatment remains a problem. The experimentally induced inhibition of gastric tone by intestinal nociceptive stimulation and chemical peritoneal stimulation involves a major sympathetic component. ${ }^{5}$ Spinovagal reflexes, however, have also been implicated mediated by the non-adrenergic non-cholinergic vagal pathway. ${ }^{6}$ The electrophysiological basis of this reflex has been identified by recording directly from vagal preganglionic fibres. ${ }^{7}$

One of the most likely candidates for this nonadrenergic non-cholinergic inhibitory transmitter in the proximal stomach of a number of species is vasoactive intestinal polypeptide (VIP) ${ }^{8-12}$ although recent evidence implicates a role also for nitric oxide at least in the rat. ${ }^{13}$ Thus there may be more than one non-adrenergic noncholinergic inhibitory transmitter regulating the gastric musculature.

In the present study we have used autoantibodies to neutralise endogenous vasoactive intestinal polypeptide as a means of investigating the role of vagal non-adrenergic non-cholinergic neurones in the recovery of gastric motility after acute surgical procedures and in gastric accommodation. Preliminary accounts of this work have been presented to the British Society of Gastroenterology ${ }^{14}$ and The 13th International Symposium on Gastrointestinal Motility. ${ }^{15}$

\section{Methods}

IMMUNISATION AND ASSAY FOR ANTI-VIP ANTIBODIES

Six ferrets were injected subcutaneously at several sites in the mid scapular region with a total of $25 \mathrm{nmol}$ equivalent of porcine vasoactive intestinal polypeptide (Bachem), conjugated with thyroglobulin and emulsified in Freund's complete adjuvant as described by Forster, Green, and Dockray. ${ }^{16}$ A similar booster injection was given four weeks after the primary injection and experiments were performed after a further two to three weeks. These animals together with eight control animals were investigated acutely under urethane anaesthesia $(1.5 \mathrm{~g} / \mathrm{kg})$ at which time arterial blood samples $(1 \mathrm{ml})$ were taken into citrated tubes for the assay of vasoactive intestinal polypeptide binding activity. The blood was spun at 4000 RPM for 20 minutes and the plasma stored at $-20^{\circ} \mathrm{C}$ for subsequent assay. The assay procedure is outlined in Figure 1.

RECORDING OF GASTRIC CORPUS MOTOR FUNCTION Gastric corpus motility was recorded manometrically as previously described. ${ }^{17}$ Briefly, experiments were performed on ferrets, fasted
Correspondence to: Dr D Grundy, Department University of Sheffield Sheffield

Accepted for publication 27 April 1992 
Assay for VIP antibodies

Plasma diluted (1:200 in phosphate buffer)

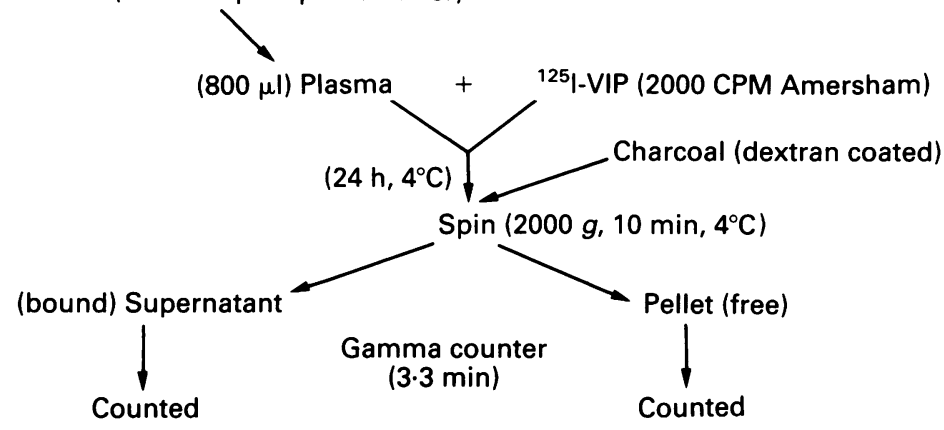

$\%$ Bound $=\frac{\text { bound }}{\text { bound }+ \text { free }} \times 100$

Figure 1: Outline of the procedure for assessing plasma vasoactive intestinal polypeptide binding activity. A blank consisting of the buffer without plasma was used to calculate non-specific binding. isotonic glycine and thereafter spontaneous corpus motility was recorded for 40 minutes in order to quantify the postsurgical recovery in gastric motility. After this time, the corpus was distended, on three occasions separated by 15 minutes, with ramp inflations at a rate of $5 \mathrm{ml} / \mathrm{min}$ up to a maximum volume of $20 \mathrm{ml}$ to determine the mean pressure rise associated with this rate and volume of distension as a reflection of the ability of the gastric corpus to accommodate fluid.

\section{DATA ANALYSIS AND STATISTICS}

Data are expressed as mean (SE) of the mean with $\mathrm{n}=$ number of animals. Statistical significance was assessed using paired and unpaired Student's $t$ test where appropriate except for the vasoactive intestinal polypeptide binding data which, because of their distribution, were assessed using the Mann-Whitney U-test. In all cases significance was taken as $\mathrm{p}<0.05$.

overnight, and anaesthetised with urethan $(1.5 \mathrm{~g} / \mathrm{kg}$ ip). After a tracheostomy, to maintain a clear airway, the jugular vein and carotid artery were cannulated for drug injection and monitoring blood pressure respectively.

At laparotomy, the greater splanchnic nerves were severed bilaterally and the stomach divided at the incisura angularis to separate the corpus and antrum. The corpus was intubated from both the antrum and from the oeosophagus; the former was connected to an Elcomatic EM-760 manometry system for the continuous recording of intracorpus pressure and the latter used to distend the corpus with up to $20 \mathrm{ml}$ isotonic glycine buffer at $\mathrm{pH} 7$ and $37^{\circ} \mathrm{C}$.

\section{PROTOCOL}

Immediately after closure of the abdominal incision, the gastric corpus was flushed with

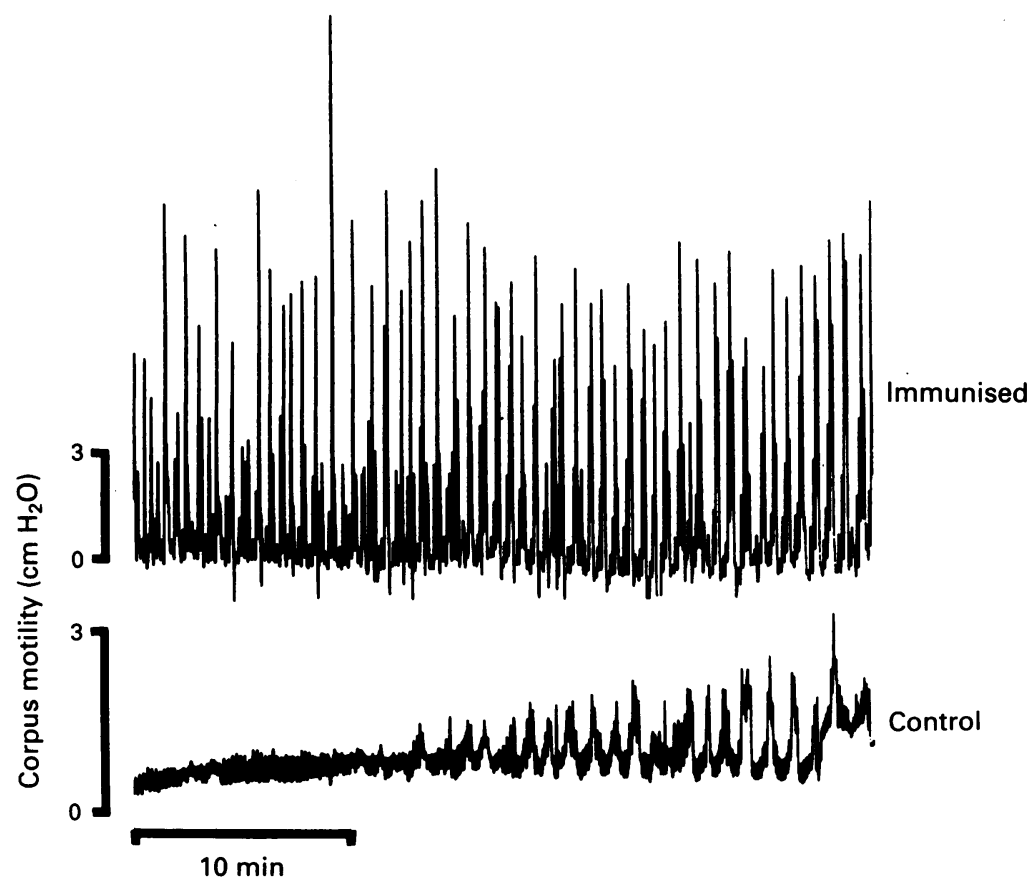

Figure 2: Intracorpus pressure during the recovery period from surgery in an immunised and a control animal to illustrate the different levels of spontaneous phasic activity. Note that the scale is different in the two traces.

\section{Results}

\section{SPONTANEOUS MOTILITY IN CONTROL AND}

IMMUNISED ANIMALS

Phasic pressure waves were taken as an index of gastric corpus contractile activity which was strikingly different in control and immunised animals. In control animals there was very little phasic activity immediately after surgery. The mean amplitude of phasic corpus contractions during the first 10 minute period after closure was $0.43(0.22) \mathrm{cm} \mathrm{H}_{2} \mathrm{O}$ and in the period 30 to 40 minutes, at the end of the recovery period, this had increased to $0.85(0.57) \mathrm{cm} \mathrm{H}_{2} \mathrm{O}(\mathrm{n}=8)$ (Figs 2, 3). In immunised animals, at a comparable time after surgery, the corpus motility was strikingly increased. The mean amplitude of phasic pressure waves at the beginning of the recording period was $7 \cdot 1(2) \mathrm{cm} \mathrm{H}_{2} \mathrm{O}(\mathrm{n}=6)$ and remained raised throughout the recovery period (Fig 3). When averaged over the whole 40 minute recording period the mean amplitude of contractions was 15 times higher in immunised animals than controls $(\mathrm{p}<0.02)$.

PRESSURE/VOLUME RELATIONSHIP IN THE GASTRIC CORPUS

The mean rise in baseline pressure in the gastric corpus during ramp inflations in control and immunised animals is shown in Figure 4. As described previously, ${ }^{17}$ the magnitude and profile of this pressure rise is a reflection of myogenic tone together with vagal cholinergic and non-adrenergic non-cholinergic reflex activity. The latter may have been attenuated by the presence of circulating vasoactive intestinal polypeptide antibodies. As can be seen in Figure 4 , however, the waveform and magnitude of the pressure rise during ramp distension in immunised and control animals was comparable. While there was a small increase in pressure at each of the $5 \mathrm{ml}$ increments in volume in immunised animals there was no significant differences between the two groups of data $(p=0 \cdot 16$ to $\mathrm{p}=0 \cdot 28)$. 


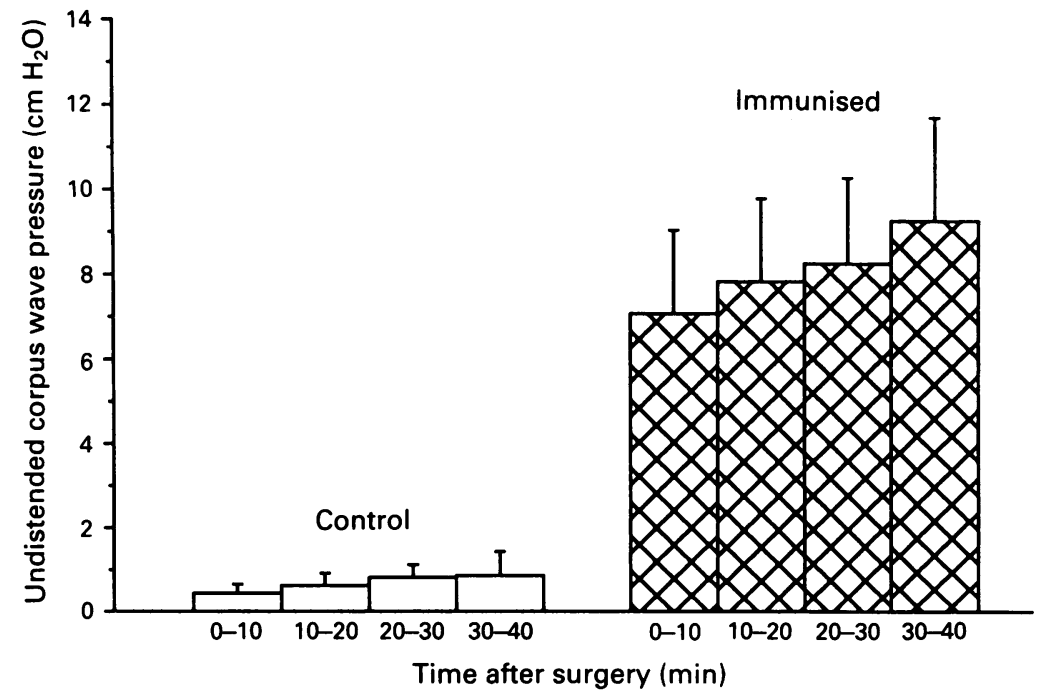

Figure 3: Mean amplitude of spontaneous phasic pressure waves in control $(n=8)$ and immunised $(n=6)$ animals during the 40 minute recovery period after surgery. In immunised ferrets there was a significant increase in the amplitude of phasic pressure waves during each of the 10 minute blocks $(p<0.02-p>0.01)$.

\section{ANTI-VIP ANTIBODIES}

The presence of circulating vasoactive intestinal polypeptide antibodies was confirmed by binding radiolabelled vasoactive intestinal polypeptide to plasma diluted $1: 200$ in phosphate buffer. The vasoactive intestinal polypeptide binding concentrations for control and immunised animals is shown in Figure 5. In control animals the mean percentage binding was $4 \cdot 1(1 \cdot 3) \%$. In immunised animals a wide range of percentage of vasoactive intestinal polypeptide binding was obtained (range $3-67 \cdot 1 \%$ ) with some overlap with control data. Nevertheless, the immunised group as a whole showed significantly higher binding than controls $(p=0.013)$. The wide range probably reflects variability in the success of the immunisation procedure. There was no significant correlation in immunised animals, however, between the percentage of vasoactive intestinal polypeptide binding and the level of spontaneous corpus motility or the pressure rise during ramp distension.

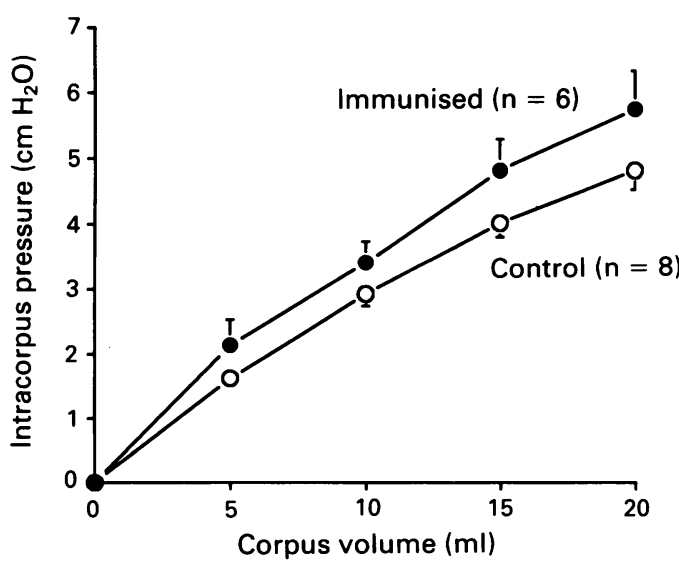

Figure 4: Pressure/volume relationship during $20 \mathrm{ml}$ ramp distension of the corpus in control and immunised animals.

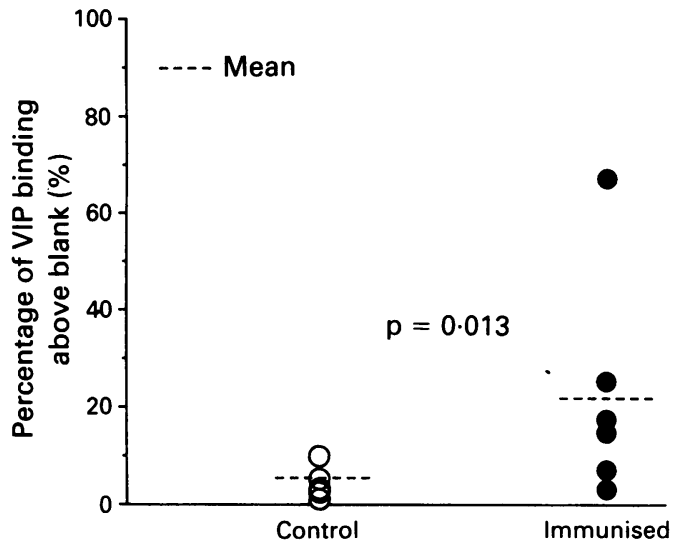

Figure 5: Plasma vasoactive intestinal polypeptide binding activity in control and immunised animals.

\section{Discussion}

A number of studies over the last decade have indicated that vasoactive intestinal polypeptide is a likely mediator of nerve induced gastric relaxation and a good candidate for the transmitter in the vagal non-adrenergic noncholinergic inhibiting pathway to the stomach. ${ }^{8-12}$ As such antisera to vasoactive intestinal polypeptide have been used in vitro to antagonise gastric relaxation caused by transmural electrical stimulation. ${ }^{810-12}$ Antigen binding fragments to vasoactive intestinal polypeptide have also been used to reversibly block field stimulation induced relaxation and inhibition of phasic activity in ferret gastric corpus strips although the effect appeared to be more marked on phasic than tonic activity. ${ }^{18}$ In vivo, immunoneutralisation of vasoactive intestinal polypeptide can reduce the inhibition of gastric emptying caused by the instillation of protein rich test meals into the duodenum. ${ }^{16}$ In the ferret, vasoactive intestinal polypeptide containing neurones have been shown to innervate the corpus and exogenous vasoactive intestinal polypeptide mimicks the effect of vagal stimulation in vivo. ${ }^{12} 18$

Antibodies raised against vasoactive intestinal polypeptide have been shown to cause catalytic cleavage of the peptide and have been used to eliminate endogenous vasoactive intestinal polypeptide mediating non-adrenergic noncholinergic relaxations in the cat trachea. ${ }^{19} \mathrm{It}$ would appear likely therefore that the rise in spontaneous corpus motility observed in the present study after immunisation is the result of hydrolysis of endogenous vasoactive intestinal polypeptide which in control animals caused marked suppression of phasic activity, especially in the immediate postoperative period. Suppression of gastric motility by noxious stimulation of the abdominal viscera is classically considered to be mediated by sympathetic mechanisms. A vagal non-adrenergic non-cholinergic component, however, has been shown in the cat. ${ }^{6}$ The present data would support the involvement of non-adrenergic non-cholinergic inhibiting nerves in the suppression of gastric motility after noxious stimulation during surgery.

While immunoneutralisation had a marked 
effect on spontaneous corpus motility there was no deficit in immunised animals in the ability of the corpus to accommodate fluid: a reflex for which non-adrenergic non-cholinergic inhibitory mechanisms are well established. ${ }^{170-22}$ It appears therefore the phasic and tonic activity of the corpus are controlled independently by different non-adrenergic non-cholinergic transmitter mechanisms. As such the present data could be interpreted as evidence against vasoactive intestinal polypeptide being the nonadrenergic non-cholinergic transmitter for reflex gastric relaxation. It is likely, however, that corpus tone is regulated by a number of cooperating mechanisms. Peptide histidine isoleucine (PHI) is derived from the same precursor as vasoactive intestinal polypeptide. It is colocalised with vasoactive intestinal polypeptide in neurones in the rat stomach and together with other C-terminal extended forms of PHI can cause gastric relaxation..$^{23}$ It has been recently suggested that nitric oxide may also be a transmitter in the non-adrenergic non-cholinergic inhibitory pathway to gastric smooth muscle. ${ }^{24}$ The rat gastric fundus appears to utilise both nitric oxide and vasoactive intestinal polypeptide as inhibitory mediators. ${ }^{13}$ Inhibition can also arise after disinhibition of the cholinergic pathways as suggested from electrophysiological studies. ${ }^{25}$ Thus while vasoactive intestinal polypeptide may primarily regulate phasic contractile activity in the gastric corpus some other transmitter(s) may regulate gastric tone. There appears to be considerable plasticity, however, in the vagal mechanisms controlling gastric tone as, there is complete recovery in the ability of the stomach to accommodate fluid after chronic vagotomy. ${ }^{26}$ In contrast, acute vagotomy causes a marked rise in pressure responses to distension as a result of the elimination of vagal inhibitory influences on gastric tone. ${ }^{172021}$ Presumably, there is some adaptation in the period after chronic vagotomy to restore relatively normal function. Similarly, the role played by vasoactive intestinal polypeptide in the regulation of gastric tone may have been superceded during the timecourse of the immunisation procedure. If this was the case, however, then a similar adaptation in the mechanisms controlling phasic activity might be expected. That this did not occur can be taken as further evidence that the inhibitory mechanisms regulating corpus tone and motility are different.

This work is supported by The Wellcome Trust. The authors are grateful to Professor G Dockray for advice on the technique of immunoneutralisation.

1 Grundy D, Schemann $M$. The neurology of the stomach. In Gustavsson S, Kumar D, Graham DY, eds. The stomach London: Churchill Livingstone, 1992: 21-40.
2 Stadaas JO. Intragastric pressure/volume relationship before and after proximal gastric vagotomy. Scand $\mathcal{f}$ Gastroenterol 1975; 10: 129-34.

3 Furness JB, Costa M. The Enteric nervous system. Edinburgh: Churchill Livingstone, 1987: 225

4 Dubois A. Mechanical gastrointestinal obstruction and paralytic ileus. In: Kumar D, Gustavsson S, eds. An illustrated guide to gastrointestinal motility. Chichester: John Wiley, guide to gastroin

5 Glise $\mathbf{H}$, Lindahl B-O, Abrahamsson $\mathrm{H}$. Reflex adrenergic inhibition of gastric motility by nociceptive intestinal stimulation and peritoneal irritation in the cat. Scand $\mathcal{f}$ Gastroenterol 1980; 15: 673-81.

6 Glise H, Abrahamsson H. Spino-vagal nonadrenergic inhibition of gastric motility elicited by abdominal nociceptive stimulation in the cat. Scand $\mathcal{f}$ Gastroenterol 1980; 15: 665-72.

7 Grundy D, Scratcherd T. A splanchno-vagal component of the inhibition of gastric motility by distension of the intestines. In: Wienbeck M, ed. Motility of the digestive tract. New York: In: Wienbeck M, ed. Motility

8 Grider JR, Rivier JR. Vasoactive intestinal peptide (VIP) as transmitter of inhibitory motor neurons of the gut: Evidence transmitter of inhibitory motor neurons of the gut: Evidence from the use of selective VIP antagonists and

F Pharmacol Exp Ther 1990; 253: 738-42.
9 Andrews PLR, Lawes INC. Characteristics of the vagally driven non-adrenergic, non-cholinergic inhibitory innervation of ferret gastric corpus. $\mathcal{F}$ Physiol (Lond) 1985; 363: $1-20$.

10 De Beurme FA, Lefebvre RA. Vasoactive intestinal polypeptide as possible mediator of relaxation in the rat gastric fundus. I Pharm Pharmacol 1988; 40: 711-5.

11 D'Amato M, De Beurme FA, Lefebvre RA. Comparison of the effect of vasoactive intestinal polypeptide and nonadrenergic non-cholinergic neurone stimulation in the cat adrenergic non-cholinergic neurone stimulation

12 Davison JS, Halliday W, Sharkey KA. Evidence in vitro for vasoactive intestinal peptide as an inhibitory transmitter in the ferret gastric corpus. [Abstract.] $\mathcal{F}$ Physiol (Lond) 1989; 409: 65 .

$13 \mathrm{Li} \mathrm{CG}$, Rand MJ. Nitric oxide and vasoactive intestinal polypeptide mediate non-adrenergic, non-cholinergi inhibitory transmission to smooth muscle of the rat gastric fundus. Eur $\mathcal{F}$ Pharmacol 1990; 191: 303-9.

14 Grundy D, Gharib Naseri MK, Hutson D. Role of the vasoactive intestinal polypeptide (VIP) in the postoperative suppression of gastric motility. [Abstract.] Gut 1990; 31: Al185.

15 Grundy D, Gharib Naseri MK, Hutson D. The effect of immuno-neutralization of VIP on gastric corpus compliance in the ferret. [Abstract.] $f$ Gastrointest Mot 1991; 3: ance 182 .

16 Forster ER, Green T, Dockray GJ. Efferent pathways in the reflex control of gastric emptying in rats. Am F Physiol 1991 260: G499-504.

17 Grundy D, Gharib-Naseri MK, Hutson D. Regulation of gastric corpus tone by the vagus nerve in the ferret. $\mathcal{f}$ Gastrointest Mot 1991; 3: 32-8.

18 Davison JS, McIntosh C, Sharkey KA. Evidence that vasoactive intestinal peptide (VIP) is an inhibitory transmitter in the ferret gastric corpus. Proc West Pharmacol Soc 1991; 34: 417-9.

19 Hakoda H, Zhouqiu X, Aizawa HH, Inoue H, Hirata M, Ito $Y$. Effects of immunization against VIP on neutrotransmission cat trachea. Am f Physiol 1991; 261: L341-8.

20 Andrews PLR, Grundy D, Lawes INC. The role of the vagus and splanchnic nerves in the regulation of intragastric pressure

21 Andrews PLR, Lawes INC. The role of vagal and intramural inhibitory reflexes in the regulation of intragastric pressur in the ferret. F Physiol (Lond) 1982; 326: 435-51

22 Andrews PLR, Lawes INC. Interactions between splanchnic and vagus nerves in the control of mean intragastric pressure in the ferret. F P hysiol (Lond) 1984; 351: 473-90.

23 Lefebvre RA, Sas S, Cauvin A. Relaxant effect of rat PHI, PHI-gly and PHV (1-42) in the rat gastric fundus. Peptides $1991 ; 12: 271-4$.

24 Boeckxstaens GE, Pelckmans PA, Bogers JJ, Bult H, De Man $\mathrm{JG}$, Oosterbosch $\mathrm{L}$, et al. Release of nitric oxide upon stimulation of nonadrenergic noncholinergic nerves in the stimulation of nonadrenergic noncholinergic nerves in the rat gastri.

25 Davison JS, Grundy D. Modulation of single vagal efferent fibre discharge by gastrointestinal afferents in the rat. f Physiol (Lond) 1978; 284: 69-82.

26 Andrews PLR, Bingham S. Adaptation of the mechanisms controlling gastric motility following chronic vagotomy in the ferret. Exp Physiol 1990; 75: 811-25. 